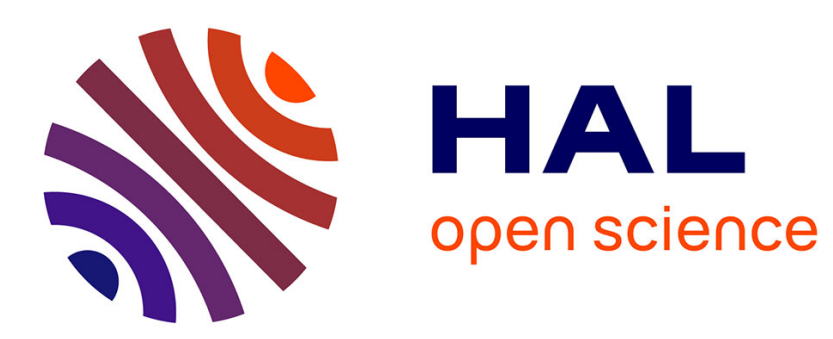

\title{
Investigating new classes of sampling sequences: Application to the stability analysis of decentralized sampled-data systems
}

\author{
Lucien Etienne, K M D Motchon, C Fiter
}

\section{- To cite this version:}

Lucien Etienne, K M D Motchon, C Fiter. Investigating new classes of sampling sequences: Application to the stability analysis of decentralized sampled-data systems. Conference on Decision and Control, Dec 2019, Nice, France. hal-02272849

HAL Id: hal-02272849

https://hal.science/hal-02272849

Submitted on 28 Aug 2019

HAL is a multi-disciplinary open access archive for the deposit and dissemination of scientific research documents, whether they are published or not. The documents may come from teaching and research institutions in France or abroad, or from public or private research centers.
L'archive ouverte pluridisciplinaire HAL, est destinée au dépôt et à la diffusion de documents scientifiques de niveau recherche, publiés ou non, émanant des établissements d'enseignement et de recherche français ou étrangers, des laboratoires publics ou privés. 


\title{
Investigating new classes of sampling sequences: Application to the stability analysis of decentralized sampled-data systems
}

\author{
L. Etienne*, K.M.D. Motchon*, C. Fiter**
}

\begin{abstract}
In this work, the stability problem of a decentralized control system where different sensors and actuators may communicate independently in an aperiodic and asynchronous manner is investigated. In order to conduct the analysis, we shift the focus from the decentralized system to the sampling sequence induced by several components communicating independently from each other. First it is shown how those sampling sequences at the level of the local component combine with each other when considering the overall system. Then the results obtained on the sampling sequence are applied, along with Lyapunov stability arguments in order to study the stability of decentralized sampled-data systems. Some experimental results obtained on an inverted pendulum benchmark are presented, to show the usefulness of the approach.
\end{abstract}

\section{INTRODUCTION}

In modern control systems where digital technologies are ubiquitous, the control task consists of the sampling of the plant outputs, the computation, and the implementation of the actuator signals. The classic way is to sample in a periodic fashion, allowing the closed-loop system to be analysed on the basis of sampled-data systems, see [1].

Complex decentralyzed sampled-data systems (either Cyber-Physical Systems (CPS) [2] or Networked Control Systems (NCS) [3]) are becoming more and more pervasive in many applications [2]. They use sensor data to determine actuation actions to physical processes. Usually, each component (sensors and actuators) is sampled, and the information is sent wirelessly to other sub-systems. Te use of the NCS/CPS paradigm enables price reduction, scalability, and ease of maintenance. In turn those architectures impose constraints on the information that can be transmitted among components both in terms of frequency and in terms of regularity. Furthermore as networks grow in size centralized/synchronized communication may become too demanding or even unrealistic. So compared with more classical control architectures, CPS/NCS can be harder to predict and require a careful investigation.

The problem of control of classical sampled-data systems has received a lot of attention in the past decade. In an environment where a micro-controller has to perform a lot of computations and where there are a lot of data transmissions, it is difficult to guarantee the periodicity of the sampling sequences (because of jitter, packet droppouts, etc.), which may lead to the system's instability (see [4] for a recent

*L.Etienne, and K. M. D. Motchon are with the URIA IMTLille-Douai. e-mail:\{lucien.etienne, djidula.motchon \}eimt-lille-douai.fr

*Christophe Fiter is with University of Lille and CRIStAL UMR CNRS 9189. e-mail:Christophe.fiter@univ-lille.fr survey on the topic). A possible way to study the stability of a sampled-data system is to use the impulsive system modelling framework and to study the stability of the resulting system [5], [6]. Hybrid models can also be very relevant to model and analyse complex behaviour of sampled-data systems [7], [8]. However in this context, sampling of the control input is supposed to be centralized/synchronized by a scheduler. In this work this assumption will be lifted.

Such a subject has recently been attracting more attention, since asynchrony in the sensors and/or actuators of sampleddata control systems may be a source of instability, just like aperiodicity. For instance, [9] considers the case of one sensor and one actuator implemented on an unsynchronized control loop while [10] studies the problem of maximal allowable clock offset between one sensor and one actuator. The work [11] considers the problem of decentralized estimation of a multi agent system in an unsynchronized setting. Using the same hybrid framework as [11], [12] shows stability of unsynchronized saturated linear systems. In [13] and [14] small gain arguments are used to establish stability of decentralized sampled-data systems while [15] use integral quadratic constraints to achieve the same objective.

The aim of this paper is to study the stability of Linear Time-Invariant (LTI) systems with several sensors and actuators that sample their signals in an aperiodic and asynchronous fashion.

In this work we shift the focus from the decentralized system to the sampling sequence induced by several components communicating independently from each other. Considering stability for entire classes of sampling sequences is not new [16] but has proven fruitful. Furthermore it is of interest to identify "good" classes of sampling sequences that naturally emerge when considering interaction of sub-components with classical sampling requirements (for instance periodic sampling.)

The paper is organized as follows. In Section II, we describe the usual main classes of events sequences and provide some important properties that will be useful in the context of decentralized control. Then, in Section III, we propose the main stability results for a general class of impulsive systems with several aperiodic and asynchronous sequences of impulses and derive some stability criterion for decentralized sampled-data control systems. Finally, in Section IV, we perform some experimentations on an inverted pendulum, to show the usefulness of the approach.

The following general notations are adopted throughout the rest of the paper:

Notation: 
- For a vector or a matrix $v, v^{\top}$ denotes its transpose.

- We define for a matrix $A, H e(A):=A+A^{\top}$.

- $\mathbb{R}^{+}$denotes the set of non-negative real numbers.

- The Cartesian product of two sets $\mathcal{S}_{1}$ and $\mathcal{S}_{1}$ is written $\mathcal{S}_{1} \times \mathcal{S}_{2}$ and for $M$ sets $\mathcal{S}_{m}, m \in\{1, \ldots, M\}$ we write $\prod_{m=1}^{M} \mathcal{S}_{m}$ for their Cartesian product (i.e. the set $\left.\left\{\omega=\left(\omega_{1}, \ldots, \omega_{M}\right) \mid \omega_{m} \in \mathcal{S}_{m}\right\}\right)$.

- For a matrix $M, \lambda_{\min }(M)$ and $\lambda_{\max }(M)$ denote its smallest and largest eigenvalues, respectively.

- For two positive definite matrices (resp positive semidefinite) $P$ and $Q$ we write $P \succ Q$ if $P-Q$ is positive definite (resp $P \succeq Q$ if $P-Q$ is positive semidefinite).

- For sets $\subset$ describes the (non strict) inclusion, while $\not \subset$ denotes the absence of non strict inclusion.

- For a set $\Omega, \operatorname{Conv}(\Omega)$ denotes its convex hull.

- Given a function $\varphi$, if its exists, $\varphi(t)^{+}$denotes the right-hand limit of $\varphi$ at the point $t$.

\section{CONSIDERED CLASSES OF EVENT SEQUENCES AND SOME IMPORTANT PROPERTIES}

This section recalls the concepts of event sequences with Min/Max/Ranged dwell time that are widely considered sampling sequences. It also shows how these classes of event sequences are connected trough each other and provides some crucial results on their sorted concatenation. Before giving formal definitions we want to point out that, qualitatively a sequence with minimal average dwell time is a sequence that can not (on average) have to many events happening on a finite interval. Such type of sequence occurs naturally when considering switched systems. For maximal average dwell time the opposite is true in the sense that events have to happen often enough. This type of sequences naturally occurs when considering sampled data stabilization of unstable plants. Ranged dwell time describe sequences where both minimum and maximum dwell time constraints have to be simultaneously verified.

\section{A. Min/Max/Ranged dwell time sequences of events}

- A sequence of events $\left\{t_{k}\right\}_{k \in \mathbb{N}}$ is said to have minimal dwell time (MinDT) $\underline{\tau}$ if $\forall k \in \mathbb{N}$ one has $t_{k+1}-$ $t_{k} \geq \tau$. The set of this class of sequences is denoted by $\mathcal{S}(\min ,[\underline{\tau}, \infty])$. Alternatively a MinDT sequence of events $\left\{t_{k}\right\}_{k \in \mathbb{N}} \in \mathcal{S}(\min ,[\underline{\tau}, \infty])$ can be defined by the following inequality: $\forall t, s \in \mathbb{R}$ with $t>s$, one has $N(t, s) \leq 1+\frac{t-s}{\tau}$, where here and in all that follows, $N(t, s)$ denotes the number of event between the instants $s$ and $t$. For such a sequence, events are not (too) close to each other. We define

$$
\mathcal{S}^{M i n D T}=\bigcup_{\underline{\tau}>0} \mathcal{S}(\min ,[\underline{\tau}, \infty]) .
$$

- A sequence of events $\left\{t_{k}\right\}_{k \in \mathbb{N}}$ is said to have an Average minimal dwell time (AMinDT) $\tau$ and a chatter bound $N$ if $\forall t, s \in \mathbb{R}, t>s$ one has: $N(t, s) \leq \underline{N}+\frac{\overline{t-s}}{\underline{\tau}}$. We denote this class of sequences by $\mathcal{S}(\min ,[\underline{\tau}, \infty], \underline{N})$. One can see that for every sequences of events $\left\{t_{k}\right\}_{k \in \mathbb{N}} \in \mathcal{S}(\min ,[\underline{\tau}, \infty], \underline{N})$, $t_{k+p}-t_{k} \geq \underline{\tau}(p+1-\underline{N})$, for all $p, k \in \mathbb{N}$. From this fact it is clear that on average, (taking a big $p$ ) the sequence is similar to the previous one. Notice that from the previous definitions one has $\mathcal{S}(\min ,[\underline{\tau}, \infty])=$ $\mathcal{S}(\min ,[\underline{\tau}, \infty], 1)$. We define

$$
\mathcal{S}^{A M i n D T}=\bigcup_{\underline{\tau}>0, \underline{N} \in \mathbb{N}} \mathcal{S}(\min ,[\underline{\tau}, \infty], \underline{N}) .
$$

- A sequence of events $\left\{t_{k}\right\}_{k \in \mathbb{N}}$ is said to have maximum dwell time (MaxDT) $\bar{\tau}$ if $\forall k \in \mathbb{N}$ one has $t_{k+1}-t_{k} \leq \bar{\tau}$. We write in this case $\left\{t_{k}\right\}_{k \in \mathbb{N}} \in$ $\mathcal{S}(\max ,[0, \bar{\tau}])$. Alternatively a MaxDT sequence of events $\left\{t_{k}\right\}_{k \in \mathbb{N}} \in \mathcal{S}(\max ,[0, \bar{\tau}])$ can be defined by the following inequality: $\forall t, s \in \mathbb{R}$ with $t>s$, one has $N(t, s) \geq-1+\frac{t-s}{\underline{\tau}}$. For such a sequence, events are not (too) distant to each other. We define

$$
\mathcal{S}^{\operatorname{Max} D T}=\bigcup_{\bar{\tau}>0} \mathcal{S}(\max ,[0, \bar{\tau}]) .
$$

- A sequence of events $\left\{t_{k}\right\}_{k \in \mathbb{N}}$ is said to have an Average maximal dwell time (AMaxDT) $\bar{\tau}$ and a chatter bound $\bar{N}$ if $\forall t, s \in \mathbb{R}, t>s$, one has $N(t, s) \geq$ $-\bar{N}+\frac{t-s}{\bar{\tau}}$. As one can see that for all $p, k \in \mathbb{N}$, $t_{k+p}-t_{k} \leq \bar{\tau}(p+1+\bar{N})$, then it is clear that on average, (taking a big $p$ ) the sequence is similar to the previous one. We denote by $\mathcal{S}(\max ,[0, \bar{\tau}], \bar{N})$ the set of a sampling sequences with average maximum dwell time $\bar{\tau}$ and chatter bound $\bar{N}$. Notice that from the previous definition of MaxDT, one has $\mathcal{S}(\max ,[0, \bar{\tau}])=$ $\mathcal{S}(\max ,[0, \bar{\tau}], 1)$. We define

$$
\mathcal{S}^{A M a x D T}=\bigcup_{\bar{\tau}>0, \bar{N} \in \mathbb{N}} \mathcal{S}(\max ,[0, \bar{\tau}], \bar{N}) .
$$

- A sequence of events with both maximum and minimum dwell time denoted ranged constrained dwell time (RDT) is defined using the following set of sequences $\mathcal{S}([\underline{\tau}, \bar{\tau}])=\mathcal{S}(\min ,[\underline{\tau}, \infty]) \cap \mathcal{S}(\max ,[0, \bar{\tau}])$, that is, $\left\{t_{k}\right\}_{k \in \mathbb{N}} \in \mathcal{S}([\underline{\tau}, \bar{\tau}])$ iff $\underline{\tau} \leq t_{k+1}-t_{k} \leq \bar{\tau}$. We define

$$
\mathcal{S}^{R D T}=\bigcup_{\bar{\tau} \geq \underline{\tau} \geq 0} \mathcal{S}([\underline{\tau}, \bar{\tau}]) .
$$

- Similarly, a sequence of events with both average maximum and average minimum dwell time denoted Average ranged constrained dwell time (ARDT) is defined using the following set of sequences:

$\mathcal{S}([\underline{\tau}, \bar{\tau}], \underline{N}, \bar{N})=\mathcal{S}(\min ,[\underline{\tau}, \infty], \underline{N}) \bigcap \mathcal{S}(\max ,[0, \bar{\tau}], \bar{N})$.

For such a sequence one has:

$$
-\bar{N}+\frac{t-s}{\bar{\tau}} \leq N(t, s) \leq \underline{N}+\frac{t-s}{\underline{\tau}} .
$$

We define

$$
\mathcal{S}^{A R D T}=\bigcup_{\bar{\tau} \geq \underline{\tau} \geq 0, \bar{N}, \underline{N} \in \mathbb{N}} \mathcal{S}([\underline{\tau}, \bar{\tau}], \underline{N}, \bar{N}) .
$$


Remark 1: The ARDT is the most general class of event sequences and all the previously defined classes can be viewed as special instances of this last class of event sequences. in particular:

- A sequence MinDT is also a sequence with AMinDT which is also a sequence with ARDT:

$$
\mathcal{S}(\min ,[\underline{\tau}, \infty])=\mathcal{S}(\min ,[\underline{\tau}, \infty], 1)=\mathcal{S}([\underline{\tau}, \infty], 1,0) .
$$

- A sequence MaxDT is also a sequence with AMaxDT which is also a sequence with ARDT:

$$
\mathcal{S}(\max ,[0, \bar{\tau}])=\mathcal{S}(\max ,[0, \bar{\tau}], 1)=\mathcal{S}([0, \bar{\tau}], 0,1) .
$$

Remark 2: The following inclusions hold:

$$
\begin{aligned}
& \mathcal{S}^{M i n D T} \subset \mathcal{S}^{A M i n D T} \subset \mathcal{S}^{A R D T}, \\
& \mathcal{S}^{M a x D T} \subset \mathcal{S}^{A M a x D T} \subset \mathcal{S}^{A R D T}, \\
& \mathcal{S}^{R D T} \subset \mathcal{S}^{A R D T} .
\end{aligned}
$$

B. Sorted concatenation of the Min/Max/Ranged dwell time sequences of events

When considering sampling sequences resulting from the integration of several components (with different timing constraints) into a system, sorted concatenation is a natural operation as it describes the sampling sequences at the level of the overall system. Therefore, this operation has properties that derive from the timing constraints of each subsystem. In turns those properties will play an important part when considering problems such as stability or performance. Consider two (non strictly) increasing sequences of events ${ }^{1}\left\{t_{k}^{1}\right\}_{k \in \mathbb{N}}$ and $\left\{t_{k}^{2}\right\}_{k \in \mathbb{N}}$, we define an operation $\diamond$ between the two sequences as: $\left\{t_{k}^{1}\right\}_{k \in \mathbb{N}} \diamond\left\{t_{k}^{2}\right\}_{k \in \mathbb{N}}=\left\{t_{k}\right\}_{k \in \mathbb{N}}$ such that $\left\{t_{k}\right\}_{k \in \mathbb{N}}$ is the (non strictly) increasing sequence whose elements belong to the union of $\left\{t_{k}^{1}\right\}_{k \in \mathbb{N}}$ and $\left\{t_{k}^{2}\right\}_{k \in \mathbb{N}}$. Figure 1 below gives a representation of the concatenated sequence $\left\{t_{k}\right\}_{k \in \mathbb{N}}$. Note that this operation is exactly a concatenation followed by a sorting.

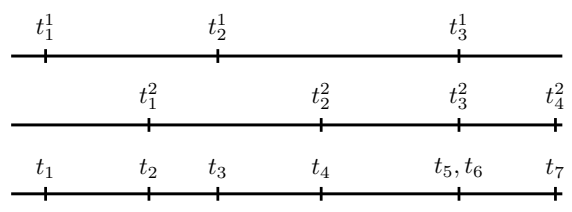

Fig. 1: Sorted concatenation of two event sequences

Given a finite number $M \in \mathbb{N}, M \geq 2$ of (non strictly) increasing event sequences $\left\{t_{k}^{m}\right\}_{k \in \mathbb{N}}, m \in\{1,2, \ldots, M\}$, their sorted concatenation is then defined by recursion as follows: $\diamond_{m=1}^{M}\left\{t_{k}^{m}\right\}=\left(\diamond_{m=1}^{M-1}\left\{t_{k}^{m}\right\}_{k \in \mathbb{N}}\right) \diamond\left\{t_{k}^{M}\right\}_{k \in \mathbb{N}}$.

Proposition 1 shows that the set of ARDT sequences of events is stable for the sorted concatenation operator (i.e. sorted concatenation of ARDT sequences is an ARDT sequence).

Proposition 1: Consider $M$ distinct series of events $\left\{t_{k}^{m}\right\}_{k \in \mathbb{N}}, m \in\{1,2, \ldots, M\}$. If for every $m \in$

\footnotetext{
${ }^{1}$ In different context, an event could be a sampling, an event triggered sampling or a switch of some discrete variable
}

$\{1,2, \ldots, M\},\left\{t_{k}^{m}\right\}_{k \in \mathbb{N}} \in \mathcal{S}\left(\left[\underline{\tau}_{m}, \bar{\tau}_{m}\right], \underline{N}_{m}, \bar{N}_{m}\right)$ with $0 \leq$ $\underline{\tau}_{m} \leq \bar{\tau}_{m} \leq \infty, \underline{N}_{m} \bar{N}_{m} \in \mathbb{N}$, then

$$
\diamond_{m=1}^{M}\left\{t_{k}^{m}\right)_{k \in \mathbb{N}} \in \mathcal{S}\left(\left[\underline{\boldsymbol{\tau}}^{\star}, \overline{\boldsymbol{\tau}}_{\star}\right], \underline{\mathbf{N}}^{\star}, \overline{\mathbf{N}}_{\star}\right)
$$

with

$$
\underline{\boldsymbol{\tau}}^{\star}=\left(\sum_{m=1}^{M} \frac{1}{\underline{\tau}_{m}}\right)^{-1}, \quad \overline{\boldsymbol{\tau}}_{\star}=\left(\sum_{m=1}^{M} \frac{1}{\bar{\tau}_{m}}\right)^{-1},
$$

and

$$
\underline{\mathbf{N}}^{\star}=\sum_{m=1}^{M} \underline{N}_{m}, \quad \overline{\mathbf{N}}_{\star}=\sum_{m=1}^{M} \bar{N}_{m} .
$$

Proof: $\left\{t_{k}^{m}\right\}_{k \in \mathbb{N}} \in \mathcal{S}\left(\left[\underline{\tau}_{m}, \bar{\tau}_{m}\right], \underline{N}_{m}, \bar{N}_{m}\right)$ is by definition equivalent to:

$$
-\bar{N}_{m}+\frac{t-s}{\bar{\tau}_{m}} \leq N_{m}(t, s) \leq \underline{N}_{m}+\frac{t-s}{\underline{\tau}_{m}},
$$

where $N_{m}(t, s)$ is the number of event of sequence $\left\{t_{k}^{m}\right\}_{k \in \mathbb{N}}$ in the time interval $[s, t]$. By definition of the sorted concatenation operator $\diamond$, the number $\tilde{N}(t, s)$ of events for the sequence $\left\{\tilde{t}_{k}\right\}_{k \in \mathbb{N}}:=\diamond_{m=1}^{M}\left\{t_{k}^{m}\right\}_{k \in \mathbb{N}}$ on $[s, t]$ is $\tilde{N}(t, s)=$ $\sum_{m=1}^{M} N_{m}(t, s)$. Therefore, one gets

$$
\sum_{m=1}^{M}\left(-\bar{N}_{m}+\frac{t-s}{\bar{\tau}_{m}}\right) \leq \tilde{N}(t, s) \leq \sum_{m=1}^{M}\left(\underline{N}_{m}+\frac{t-s}{\underline{\tau}_{m}}\right),
$$

which, according to notations (2) and (3), can be rewritten as follows

$$
-\overline{\mathbf{N}}_{\star}+\frac{t-s}{\overline{\boldsymbol{\tau}}_{\star}} \leq \tilde{N}(t, s) \leq \underline{\mathbf{N}}^{\star}+\frac{t-s}{\underline{\boldsymbol{\tau}}^{\star}},
$$

and this concludes the proof.

Corollaries 1-3 are immediate consequences of Proposition 1 and Remark 1.

Corollary 1: Let $\left\{t_{k}^{m}\right\}_{k \in \mathbb{N}}, m \in\{1,2, \ldots, M\}$ be distinct series of events such that $\left\{t_{k}^{m}\right)_{k \in \mathbb{N}} \in \mathcal{S}\left(\max ,\left[0, \bar{\tau}_{m}\right]\right)$ for every $m \in\{1,2, \ldots, M\}$. Then

$$
\diamond_{m=1}^{M}\left\{t_{k}^{m}\right\}_{k \in \mathbb{N}} \in \mathcal{S}\left(\max ,\left[0, \overline{\boldsymbol{\tau}}_{\star}\right], M\right),
$$

where $\bar{\tau}_{\star}$ is defined in (2).

Corollary 2: Let $\left\{t_{k}^{m}\right\}_{k \in \mathbb{N}}, m \in\{1,2, \ldots, M\}$ be distinct event sequences such that $\left\{t_{k}^{m}\right\}_{k \in \mathbb{N}} \in \mathcal{S}\left(\left[\underline{\tau}_{m}, \bar{\tau}_{m}\right]\right)$ for every $m \in\{1,2, \ldots, M\}$. Then

$$
\diamond_{m=1}^{M}\left\{t_{k}^{m}\right\}_{k \in \mathbb{N}} \in \mathcal{S}\left(\left[\underline{\boldsymbol{\tau}}^{\star}, \overline{\boldsymbol{\tau}}_{\star}\right], M, M\right),
$$

where constants $\underline{\tau}^{\star}$ and $\bar{\tau}_{\star}$ are given by (2).

Corollary 3: Consider two distinct series of events $\left\{t_{k}^{m}\right\}_{k \in \mathbb{N}} m \in\{1,2\}$ such that $\left\{t_{k}^{1}\right\}_{k \in \mathbb{N}} \in \mathcal{S}(\max ,[0, \bar{\tau}])$ and $\left\{t_{k}^{2}\right\}_{k \in \mathbb{N}} \in \mathcal{S}(\min ,[\underline{\tau}, \infty])$. Then

$$
\left\{t_{k}^{1}\right\}_{k \in \mathbb{N}} \diamond\left\{t_{k}^{2}\right\}_{k \in \mathbb{N}} \in \mathcal{S}([0, \bar{\tau}], 1,1) .
$$

Remark 3: For two classes of sampling sequence $\mathcal{D}$ and $\mathcal{T}$ let us denote the set of concatenated sequences

$\mathcal{D} \diamond \mathcal{T}=\left\{\left\{d_{k}\right\}_{k \in \mathbb{N}} \diamond\left\{t_{k}\right\}_{k \in \mathbb{N}} \mid\left\{d_{k}\right\}_{k \in \mathbb{N}} \in \mathcal{D},\left\{t_{k}\right\}_{k \in \mathbb{N}} \in \mathcal{T}\right\}$.

Making use of remark 1 and proposition 1 it is easy to study how classes of sampling behave when combined with the 
operation $\diamond$. Among other results we find in a straightforward manner that:

$$
\begin{aligned}
& \mathcal{S}^{M i n D T} \diamond \mathcal{S}^{M i n D T} \not \subset \mathcal{S}^{M i n D T}, \\
& \mathcal{S}^{M i n D T} \diamond \mathcal{S}^{M i n D T} \subset \mathcal{S}^{A M i n D T}, \\
& \mathcal{S}^{\operatorname{Max} D T} \diamond \mathcal{S}^{\operatorname{Max} D T} \not \subset \mathcal{S}^{\operatorname{Max} D T}, \\
& \mathcal{S}^{M a x D T} \diamond \mathcal{S}^{M a x D T} \subset \mathcal{S}^{A M a x D T}, \\
& \mathcal{S}^{M i n D T} \diamond \mathcal{S}^{M a x D T} \subset \mathcal{S}^{A R D T} \text {, } \\
& \mathcal{S}^{R D T} \diamond \mathcal{S}^{R D T} \subset \mathcal{S}^{A R D T} \text {. }
\end{aligned}
$$

\section{Discussion}

Remark 2 gives the generality of the possible sampling sets (where $\mathcal{S}^{A R D T}$ is the most general). Furthermore from remark 3 it appears that $\mathcal{S}^{A M i n D T}, \mathcal{S}^{A M a x D T}, \mathcal{S}^{A R D T}$ are stable under the operation $\diamond$. However when considering the operation $\diamond$ on different classes of sampling sequences only $\mathcal{S}^{A R D T}$ is stable. This fact highlights the importance of studying $\mathcal{S}^{A M i n D T}$ (which is a well known fact highlighted in [17],[16]) but also $\mathcal{S}^{A M a x D T}$ (which is a less known fact). A contribution of this work is to highlight the fact that for systems where each subcomponent has some RDT constraints, the important class to consider is $\mathcal{S}^{A R D T}$.

\section{Stability OF DECENTRALIZED Systems}

\section{A. Simple example: case of two MinDT sampling sequences}

Let's consider the impulsive decentralized system described by the following equations:

$$
\left\{\begin{array}{l}
\dot{x}(t)=A x(t) \quad t \geq t_{0} \in[0, \infty) \\
x\left(t_{k}^{1}\right)^{+}=J_{1} x\left(t_{k}^{1}\right), k \in \mathbb{N} \\
x\left(t_{k}^{2}\right)^{+}=J_{2} x\left(t_{k}^{2}\right), k \in \mathbb{N}
\end{array}\right.
$$

where $x(t) \in \mathbb{R}^{n}$ is the state vector, and $\left\{t_{k}^{1}\right\}_{k \in \mathbb{N}}$ and $\left\{t_{k}^{2}\right\}_{k \in \mathbb{N}}$ are two sequences of events such that $t_{0}^{1}=t_{0}^{2}=t_{0}$; $A, J_{1}$ and $J_{2}$ are matrices of appropriate dimensions. The trajectory of the system is assumed to be continuous from the left and the solution can be iteratively defined. Furthermore, it is also assumed that there exists a matrix $P=P^{\prime} \succ 0$ and two constants $\alpha>0$ and $0<\mu$ such that:

A1 $A^{\top} P+P A+\alpha P \preceq 0$,

A2 $J_{m}^{\top} P J_{m}-\mu P \preceq 0, m \in\{1,2\}$,

A3 $\left\{t_{k}^{m}\right\}_{k \in \mathbb{N}} \in \mathcal{S}\left(\min ,\left[\tau_{m}, \infty\right], N_{m}\right), m \in\{1,2\}$, with $\tau_{m}>0$ and $N_{m} \in \mathbb{N}$.

From the previous set of results one has that

$$
\left(t_{k}^{1}\right)_{k \in \mathbb{N}} \diamond\left(t_{k}^{2}\right)_{k \in \mathbb{N}} \in \mathcal{S}\left(\min ,\left[\frac{\tau_{1} \tau_{2}}{\tau_{1}+\tau_{2}}, \infty\right], N_{1}+N_{2}\right),
$$

and furthermore, one can prove the following stability result:

Proposition 2: If assumptions A1-A3 are verified and $\alpha>\frac{\ln (\mu)\left(\tau_{1}+\tau_{2}\right)}{\tau_{1} \tau_{2}}$ then system (7) is exponentially stable.

$$
\text { Proof: }
$$

The proof is similar to the one obtained when considering stability of switched system with average dwell time [18]. In what follows we will apply the proposed methodology for a more complex class of systems.
B. A general stability result for impulsive decentralized systems

Consider the impulsive decentralized system described by:

$$
\left\{\begin{array}{l}
\dot{x}(t)=A x(t), t \geq t_{0} \in[0, \infty), \\
x\left(t_{k}^{m}\right)^{+}=J_{m} x\left(t_{k}^{m}\right), \forall k, \in \mathbb{N} \forall m \in\{1,2, \ldots, M\},
\end{array}\right.
$$

where $x(t) \in \mathbb{R}^{n}$ is the state vector, and $\left\{t_{k}^{m}\right\}_{k \in \mathbb{N}}, m \in$ $\{1,2, \ldots, M\}$ are $M$ sequences of events such that $t_{0}^{m}=t_{0}$ for all $m \in\{1,2, \ldots, M\}$ and

$$
\forall m \in\{1,2, \ldots, M\}, \quad\left\{t_{k}^{m}\right\}_{k \in \mathbb{N}} \in \mathcal{S}\left(\left[\underline{\tau}_{m}, \bar{\tau}_{m}\right]\right)
$$

for some constants $0 \leq \bar{\tau}_{m} \leq \underline{\tau}_{m} \leq \infty ; A$ and $J_{m}$, $m \in\{1,2, \ldots, M\}$ are matrices of appropriate dimensions. The state trajectory of the system is also assumed to be continuous from the left.

An exponential stability result for the impulsive decentralized system (9)-(10) is provided in Theorem 1.

Theorem 1: Consider the impulsive decentralized system (9) with sampling sequences (10). If there exist symmetric matrices $P_{m} \in \mathbb{R}^{n \times n}, m \in\{1,2, \ldots, M\}$ and two constants $\alpha \in \mathbb{R}$ and $\mu \in \mathbb{R}^{+}$such that the following statements hold: $(i) \forall \tau=\left(\begin{array}{lll}\tau_{1} & \cdots & \tau_{M}\end{array}\right)^{\top} \in \mathbb{S}:=\prod_{m=1}^{M}\left\{0, \bar{\tau}_{m}\right\}$,

$$
P(\tau):=P_{0}+\sum_{m=1}^{M} \tau_{m} P_{m} \succ 0
$$

(ii) $\forall \tau \in \mathbb{S}, H e(P(\tau) A)+\sum_{m=1}^{M} P_{m}+\alpha P(\tau) \prec 0$,

(iii) $\forall m \in\{1,2, \ldots, M\}, \forall \tau \in \mathbb{S}_{m}:=\left\{\tau \in \mathbb{R}^{M}: \forall r \in\right.$ $\{1,2, \ldots, M\}, r \neq m, \tau_{r} \in\left\{0, \bar{\tau}_{r}\right\}$ and $\left.\tau_{m} \in\left\{\underline{\tau}_{m}, \bar{\tau}_{m}\right\}\right\}$,

$$
\left(\begin{array}{cc}
P\left(R_{m} \tau\right) & P\left(R_{m} \tau\right) J_{m} \\
* & \mu P(\tau)
\end{array}\right) \succ 0
$$

where $R_{m} \in \mathbb{R}^{M \times M}$ is the matrix defined by:

$$
\left(R_{m}\right)_{i j}= \begin{cases}0 & \text { if } \quad i \neq j \text { or } i=j=m, \\ 1 & \text { if } \quad i=j \neq m,\end{cases}
$$

(iv) $\alpha$ and $\mu$ satisfy

$$
\alpha \geq\left\{\begin{array}{lll}
\frac{\ln (\mu)}{\overline{\boldsymbol{\tau}}_{\star}} & \text { if } & 0<\mu \leq 1, \\
\frac{\ln (\mu)}{\underline{\boldsymbol{\tau}}^{\star}} & \text { if } & \mu>1,
\end{array}\right.
$$

with $\tau^{\star}$ and $\bar{\tau}_{\star}$ the constants defined in (2). Then system (9)-(10) is exponentially stable with a decay rate

$$
\beta=\left\{\begin{array}{lll}
\frac{1}{2}\left(\alpha-\frac{\ln (\mu)}{\overline{\boldsymbol{\tau}}_{\star}}\right) & \text { if } & 0<\mu \leq 1, \\
\frac{1}{2}\left(\alpha-\frac{\ln (\mu)}{\underline{\boldsymbol{\tau}}^{\star}}\right) & \text { if } & \mu>1 .
\end{array}\right.
$$

Proof:

Notice first that, for the sampling sequences $\left\{t_{k}^{m}\right\}_{k \in \mathbb{N}}$, $m \in\{1,2, \ldots, M\}$ satisfying (10), according to corollary 2 , their sorted concatenation $\left\{t_{k}\right\}_{k \in \mathbb{N}}=\diamond_{m=1}^{M}\left\{t_{k}^{m}\right\}_{k \in \mathbb{N}}$ verifies relation (5). Now, let $t \geq t_{0}$ be an arbitrary time instant. 
Consider the Lyapunov function $V$ defined by:

$$
V(x, s)=x^{\top} P(\tau(s)) x, \forall x \in \mathbb{R}^{n}, s \in\left[t_{0}, \infty\right),
$$

where the matrix valued function $P$ is given by (11) and

$$
\tau(s)=\left(\begin{array}{llll}
s-t_{k_{1}^{\star}}^{1}(s) & s-t_{k_{2}^{\star}}^{2}(s) & \cdots & s-t_{k_{M}^{\star}}^{M}(s)
\end{array}\right)
$$

with

$$
t_{k_{m}^{\star}}^{m}(s)=\max \left\{t_{k}^{m}: t_{k}^{m}<s\right\},
$$

the last time before $s$ that sensor $m$ sends the state's measure to the process.

For any $s \in\left[t_{0}, \infty\right)$, by construction of $\tau(s)$ in (18) and of the time instants $t_{k_{m}^{\star}}^{m}(s)$ with $m \in\{1,2, \ldots, M\}$, from the property (10) of the $M$ sampling sequences, one can see that

$$
\tau(s) \in \prod_{m=1}^{M}\left[0, \bar{\tau}_{m}\right]=\operatorname{Conv}(\mathbb{S}) .
$$

Then, from Assumption (11), by convexity, one can see that $P(\tau(s)) \succ 0$, and therefore, the function $V$ is clearly positive definite in its first variable.

Now, consider $s \in\left(t_{k}, t_{k+1}\right], k \in \mathbb{N}$. From the dynamics equation (9) of the system, we get:

$$
\begin{aligned}
\frac{\mathrm{d}}{\mathrm{d} s} V(x(s), s)=x^{\top}(s)[H e(P(\tau(s)) & A)+ \\
& \left.\frac{\mathrm{d}}{\mathrm{d} s} P(\tau(s))\right] x(s) .
\end{aligned}
$$

Then, since for any $s \in\left(t_{k}, t_{k+1}\right], k \in \mathbb{N}$, we have $\dot{\tau}(s)=$ $\left(\begin{array}{llll}1 & 1 & \cdots & 1\end{array}\right)^{\top}$, we get

$$
\frac{\mathrm{d}}{\mathrm{d} s} P(\tau(s))=\sum_{m=1}^{M} P_{m},
$$

and thus we obtain:

$$
\frac{\mathrm{d}}{\mathrm{d} s} V(x(s), s)+\alpha V(x(s), s)=x^{\top}(s) \Gamma(s) x(s),
$$

where

$$
\Gamma(s)=H e(P(\tau(s) A))+\sum_{m=1}^{M} P_{m}+\alpha P(\tau(s)) .
$$

Recalling that $\tau(s) \in \operatorname{Conv}(\mathbb{S})$, using Assumption (12) and convexity arguments we get that $\Gamma(s) \prec 0$, and therefore, from (19), we have

$$
\frac{\mathrm{d}}{\mathrm{d} s} V(x(s), s) \leq-\alpha V(x(s), s), \quad \forall s \in\left(t_{k}, t_{k+1}\right] .
$$

After integration on $\left(t_{k}, s\right]$ with $s \in\left(t_{k}, t_{k+1}\right]$, we get:

$$
V(x(s), s) \leq \mathrm{e}^{-\left(s-t_{k}\right) \alpha} V\left(x\left(t_{k}\right), t_{k}\right)^{+},
$$

where $V\left(x\left(t_{k}\right), t_{k}\right)^{+}$is the right-hand limit of $V(x(\cdot), \cdot)$ at $t_{k}$, that is, $V\left(x\left(t_{k}\right), t_{k}\right)^{+}=\lim _{s \rightarrow t_{k}, s>t_{k}} V(x(s), s)$. It follows from (26) and the definition (17) of the Lyapunov function $V$ that

$$
V\left(x\left(t_{k}\right), t_{k}\right)^{+}-\mu V\left(x\left(t_{k}\right), t_{k}\right)=x^{\top}\left(t_{k}\right) \widetilde{\Gamma}\left(t_{k}\right) x^{\top}\left(t_{k}\right)
$$

where the matrix $\widetilde{\Gamma}\left(t_{k}\right)$ is given by:

$$
\widetilde{\Gamma}\left(t_{k}\right)=J_{m_{o}}^{\top} P\left(\tau\left(t_{k}\right)\right)^{+} J_{m_{o}}-\mu P\left(\tau\left(t_{k}\right)\right)
$$

with $m_{o} \in\{1,2, \ldots, M\}$ such that $t_{k}=t_{k_{o}}^{m_{o}}$ for some $k_{o} \in$ $\mathbb{N}$. Using (18), one can see that

$$
\begin{aligned}
& \tau\left(t_{k}\right) \in\left(\prod_{m=1}^{m_{o}-1}\left[0, \bar{\tau}_{m}\right]\right) \times\left[\underline{\tau}_{m_{o}}, \bar{\tau}_{m_{o}}\right] \times \\
&\left(\prod_{m=m_{o}+1}^{M}\left[0, \bar{\tau}_{m}\right]\right)=\operatorname{Conv}\left(\mathbb{S}_{m_{o}}\right),
\end{aligned}
$$

and

$$
P\left(\tau\left(t_{k}\right)\right)^{+}=P\left(R_{m_{o}} \tau\left(t_{k}\right)\right) .
$$

Thus, by applying the Schur complement to (13), by convexity arguments, we get that $\widetilde{\Gamma}\left(t_{k}\right) \prec 0$. Consequently, the inequality

$$
V\left(x\left(t_{k}\right), t_{k}\right)^{+} \leq \mu V\left(x\left(t_{k}\right), t_{k}\right)
$$

holds. This inequality and (20) imply that for $s \in\left(t_{k}, t_{k+1}\right]$,

$$
V(x(s), s) \leq \mu \mathrm{e}^{-\left(s-t_{k}\right) \alpha} V\left(x\left(t_{k}\right), t_{k}\right) .
$$

In particular, for $s=t_{k+1}$ we have $V\left(x\left(t_{k+1}\right), t_{k+1}\right) \leq$ $\mu \mathrm{e}^{-\left(t_{k+1}-t_{k}\right) \alpha} V\left(x\left(t_{k}\right), t_{k}\right), \forall k \in \mathbb{N}$ and iterating this relation for $k \in\left\{0,1, \ldots N\left(t, t_{0}\right)\right\}$ with $N\left(t, t_{0}\right)$ the number of samplings of the concatenated sequence $\left\{t_{k}\right\}_{k \in \mathbb{N}}=$ $\diamond_{m=1}^{M}\left\{t_{k}^{m}\right\}_{k \in \mathbb{N}}$ in the time interval $\left[t_{0}, t\right)$, we get:

$$
V(x(t), t) \leq \mu^{N\left(t, t_{0}\right)} \mathrm{e}^{-\left(t-t_{0}\right) \alpha} V\left(x\left(t_{0}\right), t_{0}\right) .
$$

According to relation (5) satisfied by the concatenated sequence, we know that

$$
-M+\frac{t-t_{0}}{\overline{\boldsymbol{\tau}}_{\star}} \leq N\left(t, t_{0}\right) \leq M+\frac{t-t_{0}}{\underline{\boldsymbol{\tau}}^{\star}},
$$

and thus, we can deduce that

$$
\mu^{N\left(t, t_{0}\right)}=\mathrm{e}^{N\left(t, t_{0}\right) \ln (\mu)} \leq \mu^{\widetilde{\mathbf{M}}} \mathrm{e}^{\left(t-t_{0}\right) \frac{\ln (\mu)}{\widetilde{\boldsymbol{\tau}}}},
$$

where the constants $\widetilde{\mathbf{M}}$ and $\widetilde{\boldsymbol{\tau}}$ are given by

$$
(\widetilde{\mathbf{M}}, \widetilde{\boldsymbol{\tau}})=\left\{\begin{array}{lll}
\left(-M, \overline{\boldsymbol{\tau}}_{\star}\right) & \text { if } & 0<\mu \leq 1, \\
\left(M, \underline{\boldsymbol{\tau}}^{\star}\right) & \text { if } \quad \mu>1 .
\end{array}\right.
$$

Furthermore, one can see that

$V\left(x\left(t_{0}\right), t_{0}\right)=x^{\top}\left(t_{0}\right) P_{0} x\left(t_{0}\right) \leq \lambda_{\max }\left(P_{0}\right)\left|x\left(t_{0}\right)\right|^{2}$

and

$$
V(x(t), t) \geq \sigma_{\min }|x(t)|^{2}
$$

with $\sigma_{\min }=\min _{\tau \in \operatorname{Conv}(\mathbb{S})} \lambda_{\min }(P(\tau))>0$. Notice that the compactness of Conv $(\mathbb{S})$ in $\mathbb{R}^{M}$, the continuity of the eigenvalue function and the Weierstrass extreme value theorem ensure the existence of $\sigma_{\min }$ that is strictly positive according to Assumption (11). Finally, using inequalities (21)-(24) and the definition (16) of the constant $\beta$, we get

$$
|x(t)| \leq \sqrt{\sigma_{\min }^{-1} \lambda_{\max }\left(P_{0}\right) \mu^{\widetilde{\mathrm{M}}}} \mathrm{e}^{-\beta\left(t-t_{0}\right)}\left|x\left(t_{0}\right)\right| .
$$

Therefore, if Assumption (15) is satisfied, the system (9)(10) is exponentially stable with a decay rate $\beta$. 


\section{Stability analysis for decentralized linear sampled-data control systems}

Consider the decentralized linear sampled-data control system described by

$$
\left\{\begin{array}{l}
\dot{x}(t)=\tilde{A} x(t)+\sum_{m=1}^{M} \tilde{B}_{m} u_{m}(t), t \geq t_{0} \in[0, \infty), \\
u_{m}(t)=K_{m} x\left(t_{k}^{m}\right), \quad t \in\left(t_{k}^{m}, t_{k+1}^{m}\right], \\
x(0)=x_{0} \in \mathbb{R}^{n}, \quad m \in\{1,2, \ldots, M\}, k \in \mathbb{N},
\end{array}\right.
$$

where $x(t) \in \mathbb{R}^{n}$ is the state vector, $u_{m}(t) \in \mathbb{R}^{p_{m}}, m \in$ $\{1,2, \ldots, M\}$ is the control input sent by the $m$ th controller to the process with sampling sequence $\left\{t_{k}^{m}\right\}_{k \in \mathbb{N}}$ such that $t_{0}^{m}=t_{0}$ for all $m \in\{1,2, \ldots, M\} ; \tilde{A}, \tilde{B}_{m}$ and $K_{m}$, $m=1,2, \ldots, M$ are matrices of appropriate dimensions. The state of the system is assumed to be continuous from the left and the sampling sequences are such that (10) holds for some constants $0 \leq \bar{\tau}_{m} \leq \underline{\tau}_{m} \leq \infty, m \in\{1,2, \ldots, M\}$. Introducing the extended state variable $X$ defined by

$$
X(t)=\left(\begin{array}{llll}
x^{\top}(t) & u_{1}^{\top}(t) & \cdots & u_{M}^{\top}(t)
\end{array}\right)^{\top} \in \mathbb{R}^{n+p_{1}+\cdots+p_{M}},
$$

system (25) can be rewritten and analysed as the following impulsive decentralised system:

$\left\{\dot{X}(t)=A X(t), t \geq t_{0} \in[0, \infty)\right.$,

$\left\{X\left(t_{k}^{m}\right)^{+}=J_{m} X\left(t_{k}^{m}\right), \forall k \in \mathbb{N}, \forall m \in\{1,2, \ldots, M\}\right.$,

with $A$ and $J_{m}$ the matrices defined by:

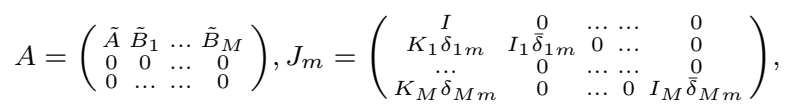

where $\delta_{i j}=\left\{\begin{array}{ll}1 & \text { if } i=j, \\ 0 & \text { otherwise, }\end{array}\right.$ and $\bar{\delta}_{i j}=1-\delta_{i j}$.

Based on this impulsive decentralized form and Theorem 1, stability of system (25) can be investigated using the following result.

Corollary 4: Consider the decentralized linear sampleddata control system (25) with event sequences $\left\{t_{k}^{m}\right\}, m \in$ $\{1,2, \ldots, M\}$ satisfying the RDT condition (10). If there exist symmetric matrices $P_{m} \in \mathbb{R}^{\left(n+p_{1}+\cdots p_{M}\right) \times\left(n+p_{1}+\cdots p_{M}\right)}$, $m \in\{1,2, \ldots, M\}$ and two constants $\alpha \in \mathbb{R}$ and $\mu \in \mathbb{R}^{+}$ such that statements $(i)-(i v)$ in Theorem 1 hold with $A$ and $J_{m}, m \in\{1,2, \ldots, M\}$ the matrices given by (27), respectively, then system (25) with sampling sequences (10) is exponentially stable with a decay rate $\beta$ given by (16).

\section{NUMERICAL EXAMPLE}

In this section, we show experimentations performed on an inverted pendulum benchmark, using the result from the previous section. The system is nonlinear, so we will use for analysis a linearisation of the model around its unstable equilibrium point.

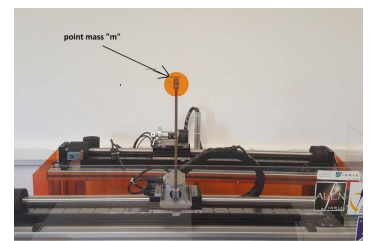

Fig. 2: Device at CRIStAL

\begin{tabular}{|c|c|}
\hline Parameters & Values \\
\hline$m_{c}$ & $3.9249 \mathrm{Kg}$ \\
\hline$m_{p}$ & $0.2047 \mathrm{Kg}$ \\
\hline$l$ & $0.2302 \mathrm{~m}$ \\
\hline$g$ & $9.81 \mathrm{~N} / \mathrm{kg}$ \\
\hline$\eta$ & $25.3 \mathrm{~N} / \mathrm{V}$ \\
\hline
\end{tabular}

TABLE I: Parameters

\section{A. System description}

The inverted pendulum (see Figure ??), consists of a cart which is driven by a motor and a pendulum. The pendulum is fixed and left free on the cart. The system has two sensors: the first one measures the linear position of the cart where the second one measures the angular position of the pendulum. Each sensor can work at a sampling periods can be as short as $1 \mathrm{~ms}$. An estimation of the linear and angular velocities is calculated using a filtered derivative. The closed-loop control has been designed on SIMULINK, and the communication between the system and the calculator (computer) is assured by a dSPACE board, using a ControlDesk software interface.

Using the linearisation of system dynamic around the upper position, one has :

$$
\left(\begin{array}{c}
\dot{x}_{p} \\
\ddot{x}_{p} \\
\dot{\theta} \\
\ddot{\theta}
\end{array}\right)=\left(\begin{array}{cccc}
0 & 1 & 0 & 0 \\
0 & 0 & \frac{-m_{p} g}{m_{c}} & 0 \\
0 & 0 & 0 & 1 \\
0 & 0 & \frac{\left(m_{c}+m_{p}\right) g}{m_{c l}} & 0
\end{array}\right)\left(\begin{array}{c}
x_{p} \\
\dot{x}_{p} \\
\theta \\
\dot{\theta}
\end{array}\right)+\left(\begin{array}{c}
0 \\
\frac{\eta}{m_{c}} \\
0 \\
\frac{-\eta}{m_{c l} l}
\end{array}\right) U .
$$

The control is designed as decentralized sampled-data linear state-feedback

$$
\begin{aligned}
& U(t)=K\left(\begin{array}{llll}
x_{p}\left(t_{k}^{1}\right) & \dot{x}_{p}\left(t_{k}^{1}\right) & \theta\left(t_{l}^{2}\right) & \left.\dot{\theta}\left(t_{l}^{2}\right)\right)^{\top},
\end{array}\right. \\
& \forall t \in\left[t_{k}^{1}, t_{k+1}^{1}\right) \cap\left[t_{l}^{2}, t_{l+1}^{2}\right), k \in \mathbb{N}, l \in \mathbb{N},
\end{aligned}
$$

with a gain $K=\left(\begin{array}{llll}5.825 & 5.883 & 24.94 & 5.140\end{array}\right)$, which was obtained using a placement of the poles $\{-100,-2+$ $2 i,-2-2 i,-2\}$.

This system can be written in the form (25) with $x=$ $\left(\begin{array}{llll}x_{p}^{\top} & \dot{x}_{p}^{\top} & \theta^{\top} & \dot{\theta}^{\top}\end{array}\right)^{\top}, M=2$,

$$
\tilde{A}=\left(\begin{array}{cccc}
0 & 1 & 0 & 0 \\
0 & 0 & \frac{-m_{p g}}{m_{c}} & 0 \\
0 & 0 & 0 \\
0 & 0 & \frac{\left(m_{c}+m_{p}\right) g}{m_{c l} l} & 0
\end{array}\right), \tilde{B}_{1}=\tilde{B}_{2}=\left(\begin{array}{c}
0 \\
\frac{\eta}{m_{c}} \\
0 \\
\frac{-\eta}{m_{c l} l}
\end{array}\right),
$$

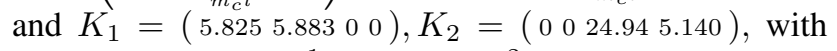
sampling sequences $\left\{t_{k}^{1}\right\}_{k \in \mathbb{N}}$ and $\left\{t_{k}^{2}\right\}_{k \in \mathbb{N}}$ satisfying (10). The evolution of the position $x_{p}$ and angular position $\theta$ is presented in Figure 4, where a large perturbation has been introduced at $t=1.6 \mathrm{~s}$, by acting on the pendulum manually. One can see that the pendulum stabilizes even in the presence of sampling intervals variations and asynchronicity of both sensors. The hectic stabilization of the pendulum can be explained by the presence of dry friction in the cart (which can be considered as a perturbation).

\section{B. Theoretical and experimental results}

Here, we have applied the results obtained in Corollary 4, with $\mu=0.999<1$ and $\alpha=-0.2$. From the proposed stability conditions, and setting lower-bounds on the sampling intervals to $\underline{\tau}_{1}=1 \mathrm{~ms}$ and $\underline{\tau}_{2}=1 \mathrm{~ms}$ 

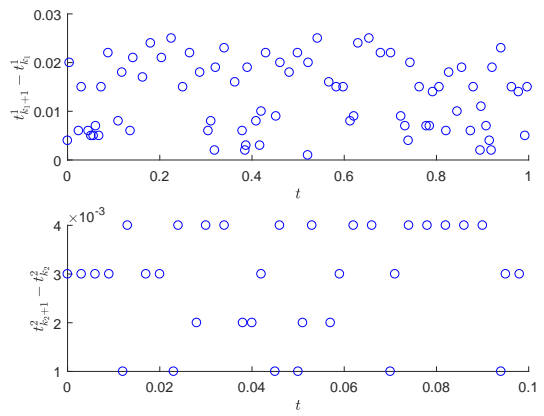

Fig. 3: Profile (zoomed) of the sampling of $x_{p}$ and $\dot{x}_{p}$ (on top between 0 and $1 \mathrm{sec}$ ), and of $\theta$ and $\dot{\theta}$ (at the bottom between 0 and $0.1 \mathrm{sec}$ )
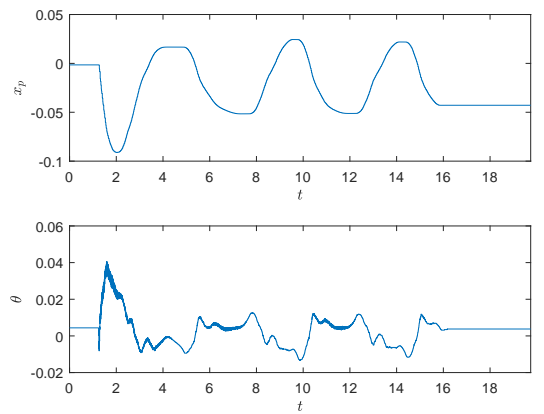

Fig. 4: Evolution of the position $x_{p}$ (top) and the angular position $\theta$ (bottom) of the inverted pendulum suject to perturbations for $\bar{\tau}_{1}=7 \mathrm{~ms}$ and $\bar{\tau}_{2}=5 \mathrm{~ms}$

(the smallest sampling period sustained by the dSPACE board), we were able to find admissible upper-bounds on the sampling intervals for each sensor. For instance, we have shown that the system is stable with upper-bounds on the sampling intervals $\bar{\tau}_{1}=25 \mathrm{~ms}$ and $\bar{\tau}_{2}=4 \mathrm{~ms}$. Therefore, from these observations, we have been able to perform some experimentations on the inverted pendulum benchmark. The results have been obtained using randomly varying sampling intervals with upper-bounds $\bar{\tau}_{1}=25 \mathrm{~ms}$ and $\bar{\tau}_{2}=4 \mathrm{~ms}$, and lower-bounds $\underline{\tau}_{1}=1 \mathrm{~ms}$ and $\underline{\tau}_{2}=1 \mathrm{~ms}$ (see Figure 3 to see the profile of the sampling intervals), satisfying the stability conditions from Corollary 4. The particular aspect of the sampling intervals for the second sensor (bottom of Figure 3), which measures $\theta$ and $\dot{\theta}$, comes from the sampling precision of the dSPACE board: since the minimal sampling interval is $\underline{\tau}_{2}=1 \mathrm{~ms}$, the maximal sampling interval is $\bar{\tau}_{2}=4 \mathrm{~ms}$, and the precision of the board's sampling is of $1 \mathrm{~ms}$, the only possible values for the sampling interval of the second sensor are $1 \mathrm{~ms}, 2 \mathrm{~ms}, 3 \mathrm{~ms}$, and $4 \mathrm{~ms}$.

\section{CONCLUSION}

In the first part of this paper we have investigated new classes of sampling sequences emerging from the sorted concatenation of well known classic sampling sequences. We have shown that maximal average dwell time and ranged average dwell time are sampling sequences worthy of more attention. This study is justified by the problem of stability of sampled-data systems where several components communicate in an asynchronous way. In the second part of this work we have used our results on sampling sequences to establish novel stability conditions of system subject to asynchronous sampled-data control.

\section{REFERENCES}

[1] K. J. Åström and B. Wittenmark, Computer-controlled systems: theory and design. Courier Corporation, 2013.

[2] P. Marwedel, "Embedded and cyber-physical systems in a nutshell," DAC. COM Knowledge Center Article, vol. 20, no. 10, 2010.

[3] J. P. Hespanha, P. Naghshtabrizi, and Y. Xu, "A survey of recent results in networked control systems," Proceedings of the IEEE, vol. 95, no. 1, pp. 138-162, 2007.

[4] L. Hetel, C. Fiter, H. Omran, A. Seuret, E. Fridman, J.P. Richard, and S.I. Niculescu, "Recent developments on the stability of systems with aperiodic sampling: an overview." Automatica, vol. 76, pp. 309-335, 2017.

[5] P. Naghshtabrizi, J. P. Hespanha, and A. R. Teel, "Exponential stability of impulsive systems with application to uncertain sampled-data systems," Systems \& Control Letters, vol. 57, no. 5, pp. 378-385, 2008.

[6] C. Briat., "Convex conditions for robust stability analysis and stabilization of linear aperiodic impulsive and sampled-data systems under dwell-time constraints," Automatica, vol. 49, no. 11, pp. 3449-3457, 2013.

[7] R. Goebel, R.G. Sanfelice, and A.R. Teel., Hybrid Dynamical Systems: modeling, stability, and robustness. Princeton University Press, 2012.

[8] L. Etienne, L. Hetel, D. Efimov, and M. Petreczky, "Observer synthesis under time-varying sampling for lipschitz nonlinear systems," Automatica, vol. 85, pp. 433-440, 2017.

[9] M. Fiacchini and I.-C. Morărescu, "Stability analysis for systems with asynchronous sensors and actuators," in 2016 IEEE 55th Conference on Decision and Control (CDC). IEEE, 2016, pp. 3991-3996.

[10] M. Wakaiki, K. Okano, and J. P. Hespanha, "Stabilization of networked control systems with clock offsets," in American Control Conference (ACC), 2015. IEEE, 2015, pp. 3522-3527.

[11] Y. Li, S. Phillips, and R. G. Sanfelice, "Robust distributed estimation for linear systems under intermittent information," IEEE Transactions on Automatic Control, vol. 63, no. 4, pp. 973-988, 2018.

[12] F. Ferrante, R. G. Sanfelice, and S. Tarbouriech, "Hybrid regional stabilization of linear systems with actuator saturation and multi-rate samplers," in 2018 European Control Conference (ECC). IEEE, 2018, pp. $1-6$.

[13] C. Fiter, T.-E. Korabi, L. Etienne, and L. Hetel, "Stability of LTI systems with distributed sensors and aperiodic sampling," in Control Subject to Computational and Communication Constraints. Springer, 2018, pp. 63-82.

[14] J. Thomas, L. Hetel, C. Fiter, N. van de Wouw, and J. Richard, " $L_{2}$-stability criterion for systems with decentralized asynchronous controllers," in 2018 IEEE Conference on Decision and Control (CDC), Dec 2018, pp. 6638-6643.

[15] M. Cantoni, M. A. Fabbro, and C.-Y. Kao, "Stability of aperiodic sampled-data feedback for systems with inputs that update asynchronously* supported in part by the australian research council (dp130104510) and national science council of taiwan (most 1052221-e-110-039-my2, most 106-2221-e-110-007-my2)." in 2018 IEEE Conference on Decision and Control (CDC). IEEE, 2018, pp. 71187123.

[16] J. P. Hespanha, "Uniform stability of switched linear systems: Extensions of lasalle's invariance principle," IEEE Transactions on Automatic Control, vol. 49, no. 4, pp. 470-482, 2004.

[17] J. P. Hespanha and A. S. Morse, "Stability of switched systems with average dwell-time," in Proceedings of the 38th IEEE conference on decision and control (Cat. No. 99CH36304), vol. 3. IEEE, 1999, pp. 2655-2660.

[18] D. Liberzon, Switching in systems and control. Springer Science \& Business Media, 2012. 